\title{
The Role of Microanalysis in Micro/Nanowire-Based Future Generation Photovoltaic Devices
}

\author{
Brent A. Wacaser,* Mark C. Reuter,* Maha M. Khayyat,** Richard Haight,* \\ Supratik Guha,* and Frances M. Ross* \\ *IBM T. J. Watson Research Center, Yorktown Heights, NY, USA \\ **King Abdul Aziz City for Science and Technology, Riyadh, Kingdom of Saudi Arabia
}

Crystalline semiconducting micro/nanowires have been grown using various precursors and fabricated by defining the nanowires in or from a crystalline material. A micro/nanowire is either a free standing crystal or a crystal that has been modified to define a one dimensional unit in which the features along two of the dimensions are on the micro/nanometer scale and the features on the other dimension are much larger. Nanowires have been proposed or used for applications including lasers, transistors, and solar cells. It has been speculated that they could present great technical and material advantages in these optoelectronic devices.[1-2] Nanowires are advantageous for use in large scale photovoltaic applications primarily because lower cost materials can be used, since the geometry of a core/shell nanowire solar cell (the core of the nanowire having a different doping than the shell or shells) may give adequate solar cell efficiencies with lower quality materials.[3] Free-standing core shell structures are desirable: these types of structure have good efficiency at possibly lower cost because the adsorption of light is good, compared to thin planar layers, and the diffusion length needed to collect carriers is much smaller as compared with thicker planar layers (see FIG. 1). In all cases, for nanowire photovoltaic devices the area of the p-n junction is larger than for similar planar devices. This means that the dark current in these devices is fundamentally higher than for planar devices with similar dimensions. The higher dark current has a negative effect on the solar cell efficiency, but it is believed that the advantages of carrier collection efficiency and/or light adsorption can outweigh this effect to make it possible to produce cost-effective nanowire based solar cells.[3]

There are three main production schemes for forming nanowires. In the top-down approach, lithography or some other means is used to pattern a planar substrate material. An anisotropic etch is then used to remove the material between the nanowires. In the bottom-up approach, either a seed particle or a patterned surface is used to define where the nanowires will grow. Physical or chemical vapor deposition (CVD) methods are then used to supply growth precursors which feed the growth of crystalline nanowires. Nanowires can also be produced by forming the crystalline nanostructures from an amorphous material.

Microscopy and microanalysis are crucial to improving and understanding micro/nanowire growth processes and solar cell performance. This talk will be laid out in three main sections. Firstly we will focus on the improvements in fabrication and understanding of the growth mechanisms that can come from in situ and ex situ analysis of nanowire growth. Secondly we will focus on the interfaces and surfaces of the nanowires and what impact they have on the solar cell device performance. These interfaces, especially the interface between the $\mathrm{p}$ and $\mathrm{n}$ areas of the solar cell diode, are critical to the quality and efficiency of solar cells.[3] Often these interfaces are difficult to control, resulting in defects (see FIG. 2) that can affect the performance of the device. Finally we will discus the 
future outlook for microanalysis of nanowire based devices. Of specific importance is the need to understand the doping and doping structure of these devices. Imaging and understanding the dopants and dopant concentration in $\mathrm{Si}$ and other semiconductor nanowires is an important field of research and there are many unanswered questions. Of special interest in this paper are the complex structures that are formed when incorporating nanoscale doping structures. Traditional doping investigation techniques like secondary ion mass spectroscopy (SIMS) are not appropriate because the structures are not planar. We will discuss current efforts in investigating this complex dopant structure, as well as some of the issues involved in the analysis. Being able to fully image and analyze these types of structured doping profiles will lead to greater understanding and help improve the control and quality of nanoscale solar cell devices.

\section{References}

[1] L. Samuelson; C. Thelander; et. al; M. W. Larsson; L. R. Wallenberg, Physica E-Low-

Dimensional Systems \& Nanostructures 2004, 25 (2-3), 313-318.

[2] H. J. Fan; P. Werner; M. Zacharias, Small 2006, 2 (6), 700-717.

[3] B. M. Kayes; H. A. Atwater; N. S. Lewis, Journal of Applied Physics 2005, 97 (11), 114302-11.
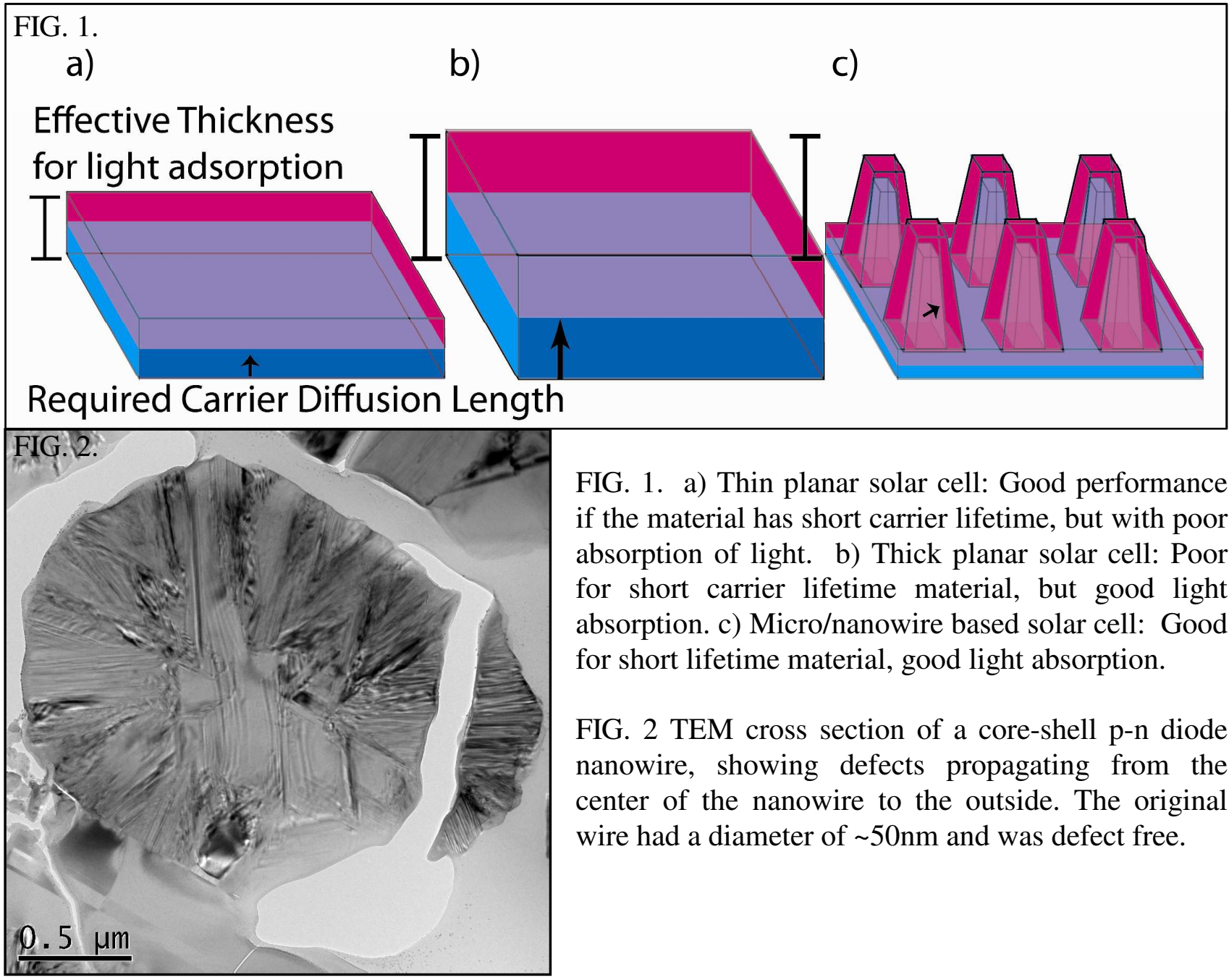

FIG. 1. a) Thin planar solar cell: Good performance if the material has short carrier lifetime, but with poor absorption of light. b) Thick planar solar cell: Poor for short carrier lifetime material, but good light absorption. c) Micro/nanowire based solar cell: Good for short lifetime material, good light absorption.

FIG. 2 TEM cross section of a core-shell $p$-n diode nanowire, showing defects propagating from the center of the nanowire to the outside. The original wire had a diameter of $\sim 50 \mathrm{~nm}$ and was defect free. 